\title{
Sindrome de Melkersson-Rosenthal: estudo clínico-patológico de um caso
}
Melkersson-Rosenthal
syndrome:
clinicopathologic
study
of one
case

$\begin{array}{lccc}\text { Lívia } & \text { Maria } & \text { Bittencourt } & \text { Nossa }^{1} \\ \text { Andrea } & \text { Leone } & \text { Costa } & \\ \text { Roberto } & \text { Lorens } \quad \text { Marback }^{2}\end{array}$

${ }^{1}$ Alunas do Curso de Especialização em Oftalmologia. Faculdade de Medicina. Universidade Federal da Bahia.

${ }^{2}$ Professor Titular de Oftalmologia. Doutor. Faculdade de Medicina. Universidade Federal da Bahia.

Endereço para correspondência: Av. Garibaldi, 1987, sala 304 - Salvador (BA) CEP 40.210-070.

\section{RESUMO}

Objetivo: Descrever os achados clínico-patológicos de um paciente com apresentação monossintomática da síndrome de Melkersson-Rosenthal. Métodos: Revisão do prontuário e das preparações histopatológicas obtidas nas biópsias. Resultados: O diagnóstico da síndrome de Melkersson-Rosenthal foi estabelecido clinicamente por meio da apresentação de edema bipalpebral unilateral. A tomografia computadorizada orbitária demonstrou expansão dos tecidos moles das pálpebras superiores. Histopatologicamente ficou evidenciada linfangite granulomatosa, com granulomas nas vizinhanças e no interior de vasos linfáticos. Conclusões: A síndrome de Melkersson-Rosenthal pode ocorrer de forma monossintomática. A demonstração histopatológica de linfangite granulomatosa confirmou o diagnóstico. A corticoterapia sistêmica foi eficiente no caso estudado.

Descritores: Síndrome de Melkersson-Rosenthal/diagnóstico; Síndrome de MelkerssonRosenthal/quimioterapia; Edema/patologia; Pálpebras; Linfangite; Tomografia computadorizada por raio-x; Corticosteróide/uso terapêutico

INTRODUÇÃO

A síndrome de Melkersson-Rosenthal é rara e caracterizada por: paralisia facial alternante, edemas recorrentes de face, pálpebras e lábios. Queilite e língua fissurada completam o quadro clínico ${ }^{(1)}$. Entretanto, recentemente novos aspectos clínicos e histopatológicos foram acrescentados à referida síndrome ${ }^{(2)}$. Alguns destes aspectos foram observados em um paciente e não foram por nós encontrados na literatura oftalmológica brasileira compulsada, o que nos estimulou a divulgá-los.

\section{RELATO DE CASO}

Paciente do sexo masculino, negro, 43 anos, registro de número 500453 do Hospital Universitário Professor Edgard Santos. História de tumefação palpebral bilateral iniciada há 8 meses, apresentando regressão espontânea pouco tempo após à esquerda. Em uso de medicações para hipertensão arterial sistêmica há cerca de 3 anos. Provas de função renal, ultra-sonografia orbitária, testes alérgicos e exames da função tireoidiana resultaram normais. A visão era igual a um em ambos os olhos. O exame oftalmológico revelou, de anormal, edema bipalpebral à direita, de consistência elástica. A tomografia das órbitas revelou imagem captante nas pálpebras superiores (Figura 1). Foi submetido, sob anestesia local, à orbitotomia anterior direita superiormente. O estudo histopatológico do material obtido revelou processo inflamatório crônico discreto, inespecífico, associado a edema. Diante da possibilidade diagnóstica de pseudotumor inflamatório linfocítico orbitário foi instituída corticoterapia sistêmica com prednisona na dose de 80 


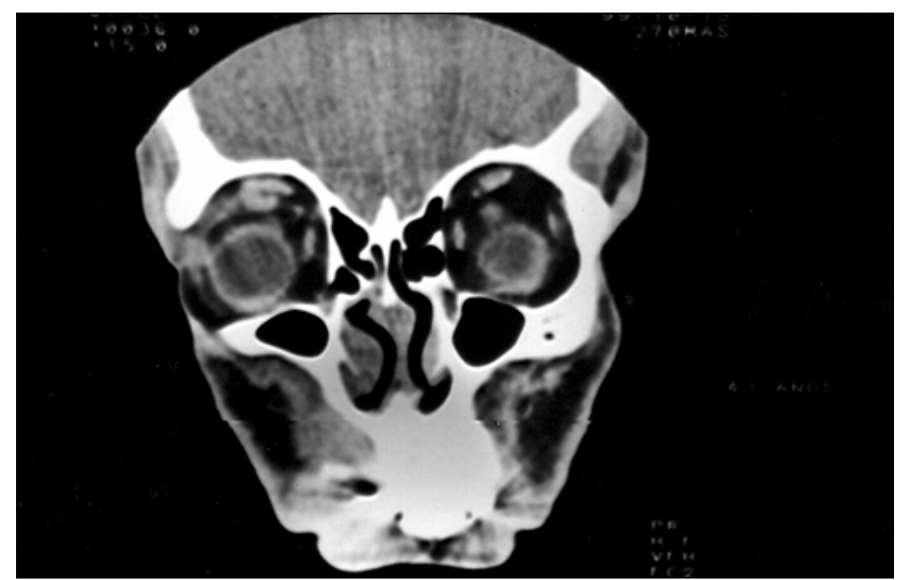

Figura 1 - Tomografia computadorizada revelou imagem captante difusa na projeção de ambas pálpebras superiores

$\mathrm{mg} / 2$ dias, $60 \mathrm{mg} / 2$ dias, $40 \mathrm{mg} / 2$ dias e manutenção de $20 \mathrm{mg} / \mathrm{dia}$ por dez dias. Ocorreu regressão do edema bipalpebral à direita. No entanto, o paciente apresentou níveis glicêmicos em torno de $500 \mathrm{mg} / \mathrm{dl}$, o que fez com que fosse suspensa a corticoterapia, levando ao ressurgimento do edema bipalpebral bilateralmente (Figura 2).

$\mathrm{Na}$ tentativa de estabelecer definitivamente o diagnóstico de pseudotumor inflamatório linfocítico e indicar radioterapia em doses antiinflamatórias foi realizada, sob anestesia local, nova biópsia de tecidos da pálpebra inferior direita, cerca de dois meses após a primeira biópsia.

O exame histopatológico revelou grande número de vasos linfáticos dilatados contendo material eosinofílico, homogêneo e acelular no seu interior. Reação inflamatória de tipo granulomatoso, constituída por linfócitos, histiócitos e células epitelióides, estava presente nas proximidades dos vasos linfáticos, e em algumas áreas, tal reação inflamatória foi vista no interior de vasos linfáticos (Figura 3). Idêntico processo granulomatoso foi também identificado na derme, nas vizinhanças do músculo orbicular e tecido adiposo pré-septal (Figura 4).

O paciente encontra-se em uso de prednisona sistêmica (20 mg/dia) associada a hipoglicemiante oral, apresentando regressão do edema de pálpebra após cerca de quatro meses.

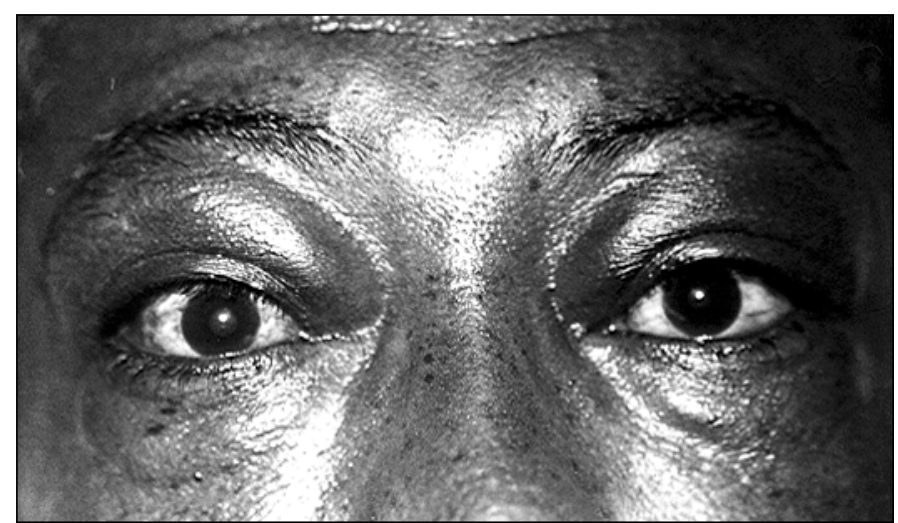

Figura 2 - Fotografia clínica. Evidencia edema bipalpebral bilateral

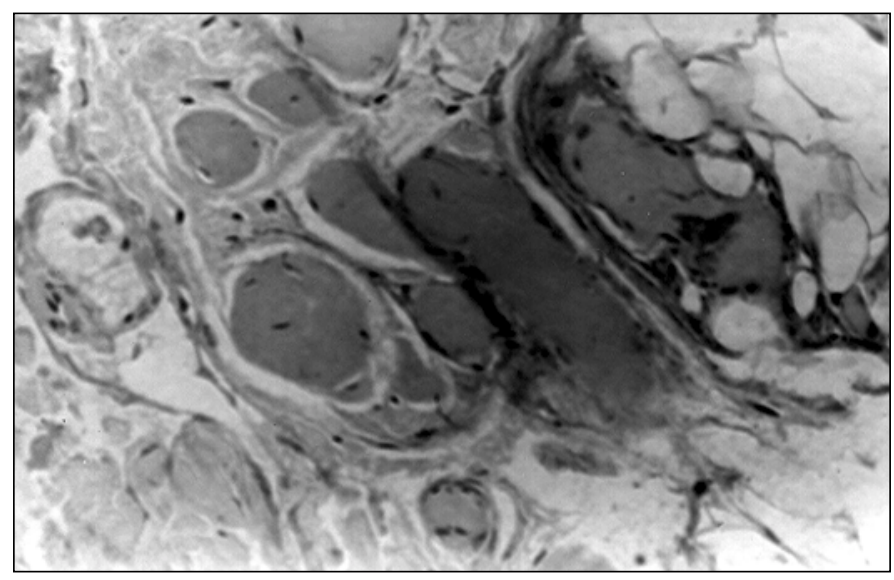

Figura 3 - Microfotografia. Vasos linfáticos dilatados contendo material eosinofílico no seu interior. Reação inflamatória composta por linfócitos, histiócitos e células epitelióides nas vizinhanças de tais vasos, e em algumas áreas, no lúmen dos mesmos (HE x 200)

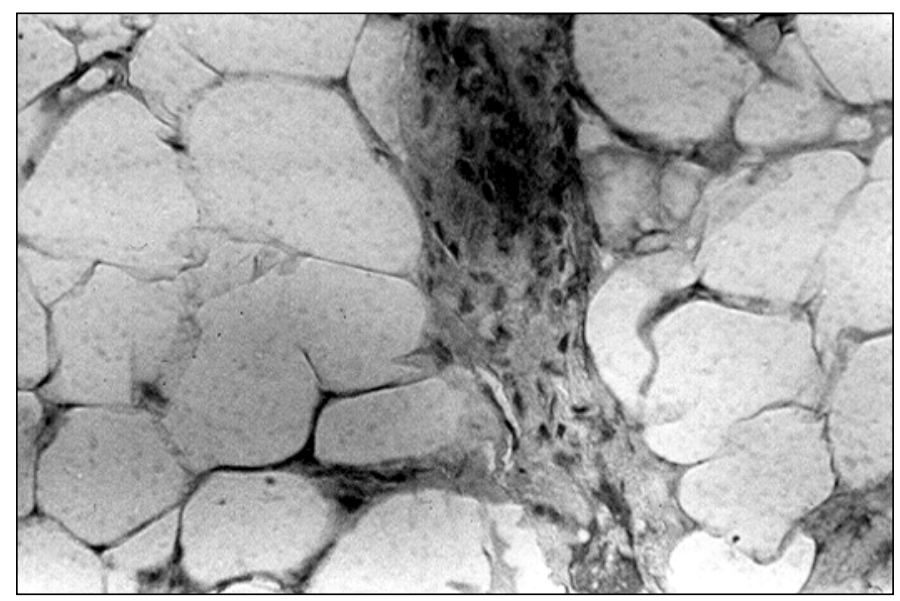

Figura 4 - Microfotografia. Semelhante reação inflamatória de tipo granulomatoso é observada no tecido adiposo pré-septal (HE x 400)

\section{DISCUSSÃO}

Paralisia e edema facial foram descritos inicialmente por Melkersson em 1928. Três anos após, Rosenthal associou a estes sintomas a língua fissurada. Esta tríade foi denominada síndrome de Melkersson-Rosenthal por Lüscher em $1949^{(3)}$. Entretanto, alguns autores advogam ser a síndrome completa rara, sendo a apresentação monossintomática ou seqüencial mais comum ${ }^{(2)}$. A incidência da síndrome de Melkersson-Rosenthal é de $0,08 \%^{(4)}$. Tem sido sugerido que o edema facial recorrente (denominado queilite granulomatosa de Meischer) $)^{(5)}$ é o mais consistente achado da síndrome de Melkerson-Rosenthal ${ }^{(1)}$, já que a paralisia de Bell e fissura lingual ( $50 \%$ na população geral) ${ }^{(6)}$ têm uma significante incidência em pacientes normais. A paralisia facial pode preceder o edema em semanas, meses ou muitos anos ${ }^{(6)}$. Outros achados oftalmológicos associados têm sido incluídos: neurite retrobulbar, lagoftalmo, opacidades corneanas, ceratoconjuntivite sicca, blefarocalase e anormalidades dos vasos retinianos ${ }^{(1-6)}$. 
Os sintomas se apresentam, geralmente, na segunda década de vida, sem prevalência de raça ou sexo ${ }^{(5)}$, embora alguns autores tenham observado uma relação de 8:1 do sexo masculino sobre o feminino ${ }^{(4)}$.

A etiologia e patogênese da síndrome de Melkersson-Rosenthal são desconhecidas: anormalidade da inervação vasomotora autonômica craniana, infecção crônica, hipersensibilidade à bactérias, granulomas dentários, alergia e herança autossômica dominante com penetrância incompleta têm sido sugeridos ${ }^{(4-5)}$.

Os achados de biópsia da mucosa bucal e palpebral têm mostrado granulomas não-caseosos compostos por células epitelióides com raras células gigantes multinucleadas. Muitos granulomas localizam-se adjacentes aos vasos linfáticos dilatados, podendo também se localizar em seus lúmens, chegando até mesmo à sua oclusão completa ${ }^{(2,4)}$. Tal aspecto histopatológico foi observado na segunda biópsia do paciente em questão.

A tomografia computadorizada pode mostrar edema dos tecidos moles orbitários ${ }^{(5)}$ ou ainda, um padrão heterogêneo destes tecidos compatível com a natureza granulomatosa do problema $^{(2)}$. No entanto, Cockerham, Hidayat, Cockerham, Depper et al. ${ }^{(2)}$ citaram que a ressonância nuclear magnética revelou, tão somente, uma expansão pré-septal não-específica. Esse fato nos leva a reservar o diagnóstico de síndrome de Melkersson-Rosenthal apenas para os pacientes que apresentem edema facial recorrente associado a achados histopatológicos compatíveis com os anteriormente citados.

Devido à raridade da síndrome de Melkersson-Rosenthal, deve-se sempre incluir no diagnóstico diferencial destes casos: dermatocalase, oftalmopatia endócrina, pseudotumor inflamatório, fenômenos alérgicos, doença de Crohn, angioedema hereditário, erisipela recorrente, sarcoidose, dermatite de contato, linfangioma e hemangioma ${ }^{(7)}$.

Variadas drogas e esquemas terapêuticos têm sido tentados como: corticóide tópico, sistêmico e intra-lesional, dapsona, clofazimina em baixa dosagem, sulfasalazina, hidroxicloroquina, penicilina, tetraciclina, eritromicina, clindamicina, difenildramina e irradiação de feixe externo ${ }^{(2,4,8-9)}$.

Glickman, Gruss, Chir, Birt et al. ${ }^{(4)}$ sugeriram um algorítmo para tratamento de pacientes com síndrome de MelkerssonRosenthal como segue abaixo:

\begin{tabular}{|c|c|c|}
\hline Sintomas & Agudo & Crônico \\
\hline $\begin{array}{l}\text { Língua } \\
\text { fissurada }\end{array}$ & Nenhum & Nenhum \\
\hline $\begin{array}{l}\text { Paralisia } \\
\text { facial }\end{array}$ & $\begin{array}{l}\text { Nenhum ou } \\
\text { esteróides }\end{array}$ & $\begin{array}{l}\text { Reanimação estática } \\
\text { dinâmica } \\
\text { Enxerto nervoso contralateral } \\
\text { Transferência de músculo livre }\end{array}$ \\
\hline \multirow[t]{2}{*}{$\begin{array}{l}\text { Edema } \\
\text { facial }\end{array}$} & $\begin{array}{l}\text { Compressas geladas } \\
\text { Esteróide sistêmico }\end{array}$ & $\begin{array}{l}\text { Lábios focal: zetaplastia } \\
\text { difuso: ressecção }\end{array}$ \\
\hline & $\begin{array}{l}\text { Esteróide local } \\
\text { Outras medidas }\end{array}$ & Pálpebras: excisão, reconstrução \\
\hline
\end{tabular}

Segundo os mesmos autores, a utilização de esteróide intra-lesional é associada com recorrência, enquanto que o esteróide sistêmico geralmente conduz à remissão.

A blefaroplastia redutora e cirurgia para descompressão do nervo facial têm sido tentadas, mas nenhuma terapia isolada ou em combinação gera remissão completa ${ }^{(2)}$, talvez pelo caráter recorrente de tal síndrome.

O paciente por nós estudado apresentou aspectos recentemente descritos na literatura ${ }^{(2)}$, ou seja: apresentação monossintomática da síndrome, imagem à tomografia computadorizada compatível com expansão de tecidos moles e finalmente à histopatologia, linfangite granulomatosa confirmando o diagnóstico.

ABS TRACT

Purpose: To describe the clinicopathological findings of a case of Melkersson-Rosenthal syndrome with monosymptomatic presentation. Methods: Review of the clinical chart and histopathological slides of the case. Results: The clinical diagnosis of MelkerssonRosenthal syndrome was suspected by the presence of unilateral bipalpebral edema. The orbital computed tomography disclosed tissue thickening of both superior lids. Granulomatous lymphangitis was demonstrated histopathologically. The granulomas were present in the neighborhood and in the lumen of the lymphatic vessels. Conclusions: Melkersson-Rosenthal syndrome can occur in a monosymptomatic presentation. The histopathological demonstration of granulomatous lymphangitis confirmed the diagnosis. Systemic steroid therapy was efficient reducing the edema of the lids.

Keywords: Melkersson-Rosenthal syndrome/diagnosis; Melkersson-Rosenthal syndrome/drug therapy; Edema/pathology; Eyelids; Lymphangitis; X-ray tomography computed; Adrenal cortex hormones/therapeutic use

\section{REFEREN C IAS}

1. May M, Galetta S. The facial nerve. In: Tasman W. editor. Duane's Clinical Ophthalmology. Philadelphia: J. B. Lippincott; 1991. v.2 p. 25-6.

2. Cockerham KP, Hidayat AA, Cockerham GC, Depper MH, Sorensen S, Cytryn AS, Gavaris PT. Melkersson-Rosenthal syndrome. Arch Ophthalmol 2000;118:227-32

3. Lüscher E. Syndrom von Melkersson-Rosenthal. Schweiz Med Wschenschr 1949;79:1-3.

4. Glickman LT, Gruss JS, Birt BD, Kohli-Dang N. The surgical management of Melkersson-Rosenthal syndrome. Plast Recontr Surg 1992;89:815-21.

5. Yeatts RP, White WL. Granulomatous blepharitis as a sign of MelkerssonRosenthal syndrome [discussion] [commented on Ophthalmology 1997;104: 1189-90]. Ophthalmology. 1997;104:1185-9.

6. Paton D. The Melkersson-Rosenthal syndrome: a case report. Am J Ophthalmol 1965;59:705-9

7. Fisher AA. Chronic lip edema with particular reference to the MelkerssonRosenthal syndrome (MRS). Cutis 1990;45:144-6.

8. Sussman GL, Yang WH, Steinberg S. Melkersson-Rosenthal syndrome. Clinical, pathologic, and therapeutic considerations. Ann Allergy 1992;69:187-94.

9. Podmore P, Burrows D. Clofazimine - an effective treatment for MelkerssonRosenthal syndrome or Miescher's cheilitis. Clin Exp Dermatol 1986;2:173-8. 